\title{
The use of health information technology in renal transplantation: A systematic review
}

DOI:

10.1016/j.trre.2021.100607

\section{Document Version}

Accepted author manuscript

Link to publication record in Manchester Research Explorer

\section{Citation for published version (APA):}

Sharma, V., Piscoran, O., Summers, A., Woywodt, A., van der Veer, S. N., Ainsworth, J., \& Augustine, T. (2021). The use of health information technology in renal transplantation: A systematic review. Transplantation Reviews, [100607]. https://doi.org/10.1016/j.trre.2021.100607

\section{Published in:}

Transplantation Reviews

\section{Citing this paper}

Please note that where the full-text provided on Manchester Research Explorer is the Author Accepted Manuscript or Proof version this may differ from the final Published version. If citing, it is advised that you check and use the publisher's definitive version.

\section{General rights}

Copyright and moral rights for the publications made accessible in the Research Explorer are retained by the authors and/or other copyright owners and it is a condition of accessing publications that users recognise and abide by the legal requirements associated with these rights.

\section{Takedown policy}

If you believe that this document breaches copyright please refer to the University of Manchester's Takedown Procedures [http://man.ac.uk/04Y6Bo] or contact uml.scholarlycommunications@manchester.ac.uk providing relevant details, so we can investigate your claim.

\section{OPEN ACCESS}




\section{Title}

The adoption of clinical risk prediction tools is limited by a lack of integration with electronic health records

\section{Authorship list}

Videha Sharma ${ }^{1,2}$

Ibrahim $\mathrm{Ali}^{3}$

Sabine N. van der Veer ${ }^{2}$

Glen P. Martin ${ }^{2}$

John Ainsworth 2

Titus Augustine ${ }^{1,4}$

\section{Affiliations}

1. Manchester University Hospitals NHS Foundation Trust, Department of Renal and Pancreatic Transplantation, Manchester Academic Health Science Centre Manchester, Greater Manchester, UK M13 9WL

2. Centre for Health Informatics, Division of Informatics, Imaging and Data Science, University of Manchester Faculty of Biology, Medicine and Health Manchester, Greater Manchester, UK M13 9PT

3. Salford Royal University NHS Foundation Trust, Department of Renal Medicine Salford, Greater Manchester, UK M6 8HD

4. University of Manchester Faculty of Biology, Medicine and Health, Division of Diabetes, Endocrinology and Gastroenterology Manchester, Greater Manchester, UK M13 9PT

\section{Corresponding Author}

\section{Mr Videha Sharma}

Department of Renal and Pancreatic Transplantation

Manchester University NHS Foundation Trust

Oxford Road, Manchester,M13 9WL

videha.sharma@postgrad.manchester.ac.uk

ORCID iD: https://orcid.org/0000-0001-7640-1239

\section{Word count}

1517
References

25

\section{Figures}

1

\section{Authors' contributions}

VS conceptualised the manuscript. VS and IA reviewed the literature and wrote the manuscript. GPM reviewed the literature and edited the manuscript. SNVDV, JA, TA reviewed and edited the manuscript. VS created the manuscript figures.

\section{Conflict of interest statement}

The authors of this manuscript declare no competing interests

\section{Funding sources}

The authors of this manuscript declare no funding sources

\section{Ethics committee approval}

Not applicable 


\section{Introduction}

Prognostic risk prediction models aim to estimate the risk of a future outcome based on available clinical parameters. ${ }^{1}$ There has been an increase in the development of such models given the move towards personalised and precision medicine, since they provide individualised risks for patients. ${ }^{2,3}$ They can help convey risks and benefits more succinctly and promote shared decision-making. Despite their benefits, risk prediction models in front-line clinical practice remain under-utilised and their potential impact on care outcomes has not been fullfilled. ${ }^{4} \mathrm{~A}$ recent systematic review of clinical decision support systems by Kwan et al published in $B M J$ demonstrated only a poor to moderate improvement of care, and highlighted the importance of designing models and tools that critically consider care processes and patient outcomes. ${ }^{5}$ Ongoing challenges include poor methodological development and lack of external validation of models. ${ }^{6,7}$ However, where robust models have been externally validated, an under-appreciated barrier to their adoption in clinical practice is the lack of integration with electronic health records (EHRs).

\section{Lack of integration as a barrier to use}

Clinical risk prediction models have clear potential to influence clinical decision-making and enhance the quality of care delivered to patients. ${ }^{8,9}$ However, developing a successful model is a rigorous process with many pitfalls, such as incomplete training data, risk of bias and failure to address clinical need. There are further challenges to externally validate and calibrate a model across different patient groups before being accepted for clinical use.$^{10}$ As a result, although there is a large body of literature on the development of risk prediction models, the evidence of successful clinical adoption and impact on care outcomes is largely absent. ${ }^{11}$

Risk prediction models are primarily developed using routinely collected clinical data, increasingly retrieved from EHRs. ${ }^{12,13}$ Thus, the variables selected and assessed during model development are those available in electronic data repositories, such as demographics, diagnostic results, medical history or drug history. Some models that were robustly validated and gained international recognition, were converted to online tools and made available through web-interfaces or mobile applications. An example of such a model is the CHA(2)DS(2)VASc score, which is used to predict the risk of stroke in patients with atrial fibrillation (AF) and thus guide anticoagulation. ${ }^{14}$ It has successfully achieved clinical impact and is the gold-standard risk prediction model for AF management as recommended by the National Institute of Health and Care Excellence. ${ }^{15}$ To use the model however, a healthcare professional is required to access a website or open an app, manually complete data fields with the patients' details and receive a risk score to guide clinical decision-making. Though this task may seem trivial compared to the potential added benefit of greater quality decision-making, the practicalities and time-constraints of clinical practice form a significant barrier to usage. This is compounded with the potential of manual transcription errors, which form a hazard of receiving incorrect results.

Another example of this is the kidney failure risk equation (KFRE) developed by Tangri et al, which is similarly available as an online tool. ${ }^{16}$ This model uses routinely collected clinical data including patient age, gender, estimated glomerular filtration rate and urinary albumin:creatinine ratio, to provide a two and five-year risk of progression to kidney failure for patients with chronic kidney disease. The KFRE has been validated internationally, and is generally reviewed positively. ${ }^{17}$ However, its widespread adoption is limited by the dependence on externally accessing the tool online and manually transcribing the variables into data fields before a risk score is presented. This impractical process has been shown to contribute as a barrier to clinical impact in primary care settings. ${ }^{18}$

A number of initiatives have attempted to increase the usability of risk prediction tools by developing userfriendly interfaces. An example of this is MDCalc@ , which is a medical calculator available via a website and a mobile application. By making the content easy to navigate and using an intuitive visual design it aims to enhance the user experience. However, the fundamental barrier of accessing the interface as an external application and manually completing fields is yet to be overcome. This is particularly a challenge as many healthcare institutions still lack interoperable EHRs and store clinical data across multiple digital systems. This means that a healthcare professional wishing to use a risk prediction tool may have to access multiple electronic sources to gather the required data to complete the fields and obtain a risk.

As healthcare providers increasingly turn to unified EHRs, the success of risk prediction models will be dependent on the integration of tools within these systems. Usability barriers may be mitigated if clinicians can access risk prediction tools, pertinent to their practice, within their local EHR and have a risk score presented automatically as fields are populated with relevant data from within the system. This intuitively simple concept would create a paradigm shift for the practical daily use of such tools and translate to patient benefit (Fig. 1). Risks may be presented graphically over a period of time to illustrate the impact of risk-addressing therapies 
and thus promote compliance. By improving accessibility in this way, it will also have an impact on future academic research evaluating these tools' performance and impact on clinical outcomes. Currently, research into usability of risk prediction tools, as standalone interfaces, or within EHRs is largely absent. User-experience is a significant part of successful product development in areas outside of healthcare and formal methodology for evaluation in other fields has been established. Recognising the importance of this as part of model development is crucial to achieve value out of future solutions. ${ }^{19}$

\section{Future concepts}

For risk prediction models that have undergone rigorous validation and assessment of clinical impact, integrating tools into EHRs will likely overcome a major barrier to use. Unfortunately, this practical implication has not been widely explored and new tools continue to appear as web-interface solutions risking non-adoption and thus failure to impact care. An example of such a recent model is the iPREDICTLIVING (2019) developed to predict risks around kidney donation to better inform renal transplant decision-making. ${ }^{20}$ In the context of a sensitive and complex clinical decision as kidney donation, detracting the clinician from the human interaction by a timeconsuming on-screen process will likely impact the patient experience. Digital health interventions should be centred around improving the quality of care delivered to patients, which includes better decisions, but also enhancing the patient-clinician relationship by providing clinicians the time to consult patients.

To realise a more streamlined workflow, a change in how we think about clinical risk prediction models is required. Front-line usability should be part of the initial exploration of the proposed model. This means involving clinicians (end-users) at the outset as part of research projects to understand how the tool would be practically used and the impact it would have on clinical encounters. The usability of such interventions plays a crucial role in preventing clinician fatigue and improving uptake..$^{21}$

Technical challenges revolve around non-standardised coding of health data across EHR providers. ${ }^{22}$ This means that even if a risk prediction model is made available as a standalone software, which can be integrated, misaligned clinical terminology may limit implementation. Involving EHR vendors early in the development of risk prediction models and imploring greater alignment across the industry will mitigate barriers to implementation and subsequent scale-up of novel solutions. An example of successful tool integration is QRISK®, which has been embedded within a number of primary care clinical management systems. ${ }^{23}$ The tool calculates individual cardiovascular risk and generates a score based on existing data. Not only has this impacted positively on front-line practice, regular use provides evolving data quality and completeness reflecting the changing population characteristics over time. This has allowed researchers to update and calibrate the tool for long-term accuracy. ${ }^{24}$ Similar implementation through hospital EHR vendors may bring such models into routine secondary care settings unifying and standardising practice. Another relevant example was the PREDICT software used in general practice in New Zealand, which automatically recorded patients' risk profiles for cardiovascular disease and prospectively linked this to coded hospital and mortality databases. This allowed a risk prediction model to be developed that took in to account an area-based deprivation index and self-reported ethnicity alongside clinical parameters, resulting in greater personalised risk profiles for individual patients. The strength of this study its prospective nature and ability to seamlessly collect healthcare data without additional intervention by clinicians delivering care. ${ }^{25}$

The tremendous potential of clinical risk prediction models mandates policy-makers to establish regulations to standardise the integration of tools into EHRs. Strategies to achieve this may be through EHR vendors working directly with data scientists to incorporate statistical models within their user interface, or alternatively provide non-proprietary application programming interfaces (APIs) for third party developers to seamlessly integrate with. The potential success of this however, heavily relies on the engagement of front-line healthcare professionals who can provide the clinical context and workflow that a risk prediction model is intending to influence. Encouraging multi-disciplinary research and development teams, which can appreciate the different facets of clinical context, statistical modelling and implementation science, supported by EHR vendors working to unified standards has the potential to bridge the current bench-to-bedside gap for clinical risk prediction models. 
1. Steyerberg EW. Clinical prediction models: Springer; 2019.

2. Banning M. A review of clinical decision making: models and current research. Journal of clinical nursing 2008; 17(2): 187-95.

3. Croskerry P. Achieving quality in clinical decision making: cognitive strategies and detection of bias. Academic Emergency Medicine 2002; 9(11): 1184-204.

4. Ahmed I, Debray TP, Moons KG, Riley RD. Developing and validating risk prediction models in an individual participant data meta-analysis. BMC medical research methodology 2014; 14(1): 3.

5. Kwan JL, Lo L, Ferguson J, et al. Computerised clinical decision support systems and absolute improvements in care: meta-analysis of controlled clinical trials. bmj 2020; 370.

6. Wynants L, Van Calster B, Bonten MM, et al. Prediction models for diagnosis and prognosis of covid-19 infection: systematic review and critical appraisal. bmj 2020; 369.

7. Collins GS, de Groot JA, Dutton S, et al. External validation of multivariable prediction models: a systematic review of methodological conduct and reporting. BMC medical research methodology 2014; 14(1): 40.

8. Raghupathi W, Raghupathi V. Big data analytics in healthcare: promise and potential. Health Inf Sci Syst 2014; 2: 3.

9. Topol E. The Topol review: preparing the healthcare workforce to deliver the digital future. Health Education England 2019.

10. Chen L. Overview of clinical prediction models. Annals of Translational Medicine 2020; 8(4).

11. Dekker FW, Ramspek CL, van Diepen M. Con: Most clinical risk scores are useless. Nephrology Dialysis Transplantation 2017; 32(5): 752-5.

12. Goldstein BA, Navar AM, Pencina MJ, loannidis J. Opportunities and challenges in developing risk prediction models with electronic health records data: a systematic review. Journal of the American Medical Informatics Association 2017; 24(1): 198-208.

13. Rothman B, Leonard JC, Vigoda MM. Future of electronic health records: implications for decision support. Mount Sinai Journal of Medicine: A Journal of Translational and Personalized Medicine 2012; 79(6): 757-68.

14. Lip GY, Nieuwlaat R, Pisters R, Lane DA, Crijns HJ. Refining clinical risk stratification for predicting stroke and thromboembolism in atrial fibrillation using a novel risk factor-based approach: the euro heart survey on atrial fibrillation. Chest 2010; 137(2): 263-72.

15. National Institute of Health and Care Excellence (2014) Clinical guideline 180 (CG180): Atrial fibrillation: management

16. Tangri N, Stevens LA, Griffith J, et al. A predictive model for progression of chronic kidney disease to kidney failure. Jama 2011; 305(15): 1553-9.

17. Peeters MJ, van Zuilen AD, van den Brand JA, et al. Validation of the kidney failure risk equation in European CKD patients. Nephrology Dialysis Transplantation 2013; 28(7): 1773-9.

18. Major RW, Shepherd D, Medcalf JF, Xu G, Gray LJ, Brunskill NJ. The Kidney Failure Risk Equation for prediction of end stage renal disease in UK primary care: An external validation and clinical impact projection cohort study. PLoS medicine 2019; 16(11): e1002955.

19. Vermeeren AP, Law EL-C, Roto V, Obrist M, Hoonhout J, Väänänen-Vainio-Mattila K. User experience evaluation methods: current state and development needs. Proceedings of the 6th Nordic conference on human-computer interaction: Extending boundaries; 2010; 2010. p. 521-30.

20. Haller MC, Wallisch C, Mjøen G, et al. Predicting donor, recipient and graft survival in living donor kidney transplantation to inform pretransplant counselling: the donor and recipient linked iPREDICTLIVING tool-a retrospective study. Transplant International 2020.

21. Sutton RT, Pincock D, Baumgart DC, Sadowski DC, Fedorak RN, Kroeker KI. An overview of clinical decision support systems: benefits, risks, and strategies for success. NPJ Digital Medicine 2020; 3(1): 1-10.

22. D'Amore JD, Mandel JC, Kreda DA, et al. Are meaningful use stage 2 certified EHRs ready for interoperability? Findings from the SMART C-CDA collaborative. Journal of the American Medical Informatics Association 2014; 21(6): 1060-8.

23. Hippisley-Cox J, Coupland C, Vinogradova $Y$, et al. Predicting cardiovascular risk in England and Wales: prospective derivation and validation of QRISK2. Bmj 2008; 336(7659): 1475-82. 
24. Hippisley-Cox J, Coupland C, Brindle P. Development and validation of QRISK3 risk prediction algorithms to estimate future risk of cardiovascular disease: prospective cohort study. bmj $2017 ; 357$ : j2099.

25. Pylypchuk R, Wells S, Kerr A, et al. Cardiovascular disease risk prediction equations in 400 000 primary care patients in New Zealand: a derivation and validation study. The Lancet 2018; 391(10133): 1897-907. 